\title{
Editorial
}

\section{Acquisition in Premature Infants Fed Human Milk: Reason to Worry?}

\section{Richard J. Schanler, MD}

Journal of Perinatology (2005) 25, 297-298. doi:10.1038/sj.jp.7211281

In its policy statement on the use of human milk, The American Academy of Pediatrics acknowledges that human milk is beneficial in the management of premature infants. ${ }^{1}$ The beneficial effects relate to improvements in host defense, absorption of nutrients, gastrointestinal function, neurodevelopmental outcomes, and the net effect of these benefits is a reduction in the economic burden imposed on the long hospitalization of the premature infant. Nevertheless, studies indicating that premature infants acquire cytomegalovirus (CMV) from human milk have become a concern for neonatologists and parents. Yet, a thorough risk/benefit analysis has yet to be conducted.

In this month's issue, Miron et al. ${ }^{2}$ report a 5.7\% rate of CMV acquisition in premature infants fed their seropositive mothers' milk. However, this rate is not significantly different from the rate of $\mathrm{CMV}$ acquisition in premature infants fed their seronegative mothers' milk or formula ( $95 \%$ confidence interval, 0 to $11 \%$ ). of those infants acquiring CMV, only one had clinical deterioration, two had mild laboratory abnormalities, and one was asymptomatic. The study is remarkable in that infants were fed fresh, not frozen, milk and that long-term follow-up data were reported. Importantly, no abnormalities in hearing or developmental examinations to 24 months were observed in those infants acquiring CMV in the postnatal period.

So, why the concern among neonatologists? Our attention was drawn to CMV and mother's milk feedings in premature infants as a result of a large series of publications reporting increased CMV acquisition postnatally in premature infants fed their CMVseropositive mother's milk fresh, not frozen. ${ }^{3,4}$ Although the acquisition rate was nearly $25 \%$, only few of the infants were symptomatic of infection. Postnatal CMV acquisition in premature infants occurred after about a month of age and usually manifested as "asymptomatic," but occasionally infants were observed to have a mild clinical decompensation, a sepsis-like

Schneider Children's Hospital at North Shore, North Shore University Hospital, Manhasset, NY, USA; and Albert Einstein College of Medicine, Bronx, NY, USA.

Address correspondence and reprint requests to Richard J. Schanler, MD, Division of NeonatalPerinatal Medicine, North Shore University Hospital, 300 Community Drive, Manhasset, NY 11030, USA. syndrome, which rarely required respiratory support. Indeed, the initial report of such deterioration failed to emphasize that similar nonspecific clinical deterioration episodes were just as common in premature infants fed CMV-free milk, 14/38 vs 16/29 infants, fed CMV-free vs CMV-positive milk, respectively, $p=0.11{ }^{4}$ Thus, despite viral or CMV-DNA acquisition being greater in premature infants fed CMV-positive milk, there does not appear to be any greater number of episodes of clinical deterioration in those infants.

Thus, current study and that reported previously both indicate that CMV acquisition in mother's milk-fed premature infants is not associated with significantly increased symptomatology.

Moreover, potential long-term effects of viral or CMV-DNA acquisition in the postnatal period have been assessed. The current study indicates that there are no long-term sequelae, at least to 24 months. Recently, follow-up of the original CMV-exposed cohort of premature infants to 2 to 4.5 years has been reported in comparison with matched control infants. ${ }^{5}$ No long-term developmental or hearing impairment was identified in this cohort of infants fed CMV-positive milk in the postnatal period.

Therefore, as neonatologists, do we need to worry about CMV acquisition from mother's milk? The "clinical deterioration" suggested (but not proven statistically) by these studies appears to have no long-term implications. Do we trade the benefits of human milk (reduced sepsis and necrotizing enterocolitis by as much as $50 \%$ ) for the 0 to $25 \%$ chance of "clinical deterioration?" The beneficial effects of human milk have been identified in premature infants fed combinations of fresh and/or frozen milk. ${ }^{6}$

Differences in CMV acquisition from fresh or frozen milk have been suggested. Yasuda's study identified a 10\% CMV acquisition rate (3/30 infants) in premature infants, all of whom were asymptomatic and all were fed mother's milk that had been frozen for a variable period of time at $-20^{\circ} \mathrm{C}$. Recently, short-term hightemperature pasteurization techniques were found to prevent CMV transmission more effectively than freezing. ${ }^{8}$

In a recent study of feeding strategies in extremely premature infants born less than 30 weeks' gestation, we found that of 69 mothers, 74\% were CMV-seropositive, but none of the 92 infants born to these 69 mothers had CMV isolated from their urine from 2 weeks to 2 months postnatal age. I would still use mother's milk as I have, fresh if available, but knowing that most milk will be frozen. The Miron study ${ }^{2}$ provides further support for this management choice. 


\section{References}

1. American Academy of Pediatrics. Section on Breastfeeding. Breastfeeding and the use of human milk. Pediatrics 2005;115:496-506.

2. Miron D, Brosilow S, Felszer K, et al. Incidence and clinical manifestations of breast milk acquired Cytomegalovirus infection in low birth weight infants. J Perinatol 2005;25:299-303.

3. Vochem M, Hamprecht K, Jahn G, Speer CP. Transmission of cytomegalovirus to preterm infants through breast milk. Pediatr Infect Dis J 1998;17:53-8.

4. Hamprecht K, Maschmann J, Vochem M, Dietz K, Speer CP, Jahn G. Epidemiology and transmission of cytomegalovirus from mother to preterm infant by breastfeeding. Lancet 2001;357:513-8.
5. Vollmer B, Seibold-Weiger K, Schmitz-Salue C, et al. Postnatally acquired cytomegalovirus infection via breast milk: effects on hearing and development in preterm infants. Pediatr Infect Dis J 2004;23:322-7.

6. Schanler RJ, Shulman RJ, Lau C. Feeding strategies for premature infants: beneficial outcomes of feeding fortified human milk vs preterm formula. Pediatrics 1999;103:1150-7.

7. Yasuda A, Kimura H, Hayakawa M, et al. Evaluation of cytomegalovirus infections transmitted via breast milk in preterm infants with a real-time polymerase chain reaction assay. Pediatrics 2003;111:1333-6.

8. Hamprecht K, Maschmann J, Muller D, et al. Cytomegalovirus (CMV) inactivation in breast milk: reassessment of pasteurization and freezethawing. Pediatr Res 2004;56:529-35. 